\title{
GFAP expression in injured astrocytes in rats
}

\author{
SHUQUAN ZHANG ${ }^{1}$, MINFEI WU $^{2}$, CHUANGANG PENG $^{2}$, GUANJIE ZHAO $^{3}$ and RUI GU \\ ${ }^{1}$ Department of Orthopedics, Tianjing Nankai Hospital, Tianjin 300100; ${ }^{2}$ Department of Orthopedics, \\ The Second Hospital of Jilin University, Changchun, Jilin 130041; Departments of ${ }^{3}$ Medicine and ${ }^{4}$ Orthopedics, \\ China-Japan Union Hospital of Jilin University, Changchun, Jilin 130033, P.R. China
}

Received September 12, 2016; Accepted February 10, 2017

DOI: $10.3892 /$ etm.2017.4760

\begin{abstract}
Glial fibrillary acidic protein (GFAP) is one of the best markers for the activation of astrocytes (AS) following injury or stress in the central nervous system (CNS). The purpose of this study was to examine the expression of GFAP and $14-3-3 \varepsilon$ in rat AS subjected to hypoxia. We established primary cultures of AS from cerebral cortex of neonatal Sprague-Dawley rats as a model of glucose deficiency and hypoxia/ischemia-reperfusion. We analyzed the activated astrocyte markers GFAP and $14-3-3 \varepsilon$ by western blot analysis and found that both increased over time, starting at $4 \mathrm{~h}$ and reaching the highest level at $72 \mathrm{~h}$, at the end of the experiment. GFAP and $14-3-3 \varepsilon$ protein localization by double-labeling immunofluorescence showed elevated expression and co-localization in the cytoplasm of AS. GFAP and 14-3-3 $\varepsilon$ expression remained elevated in AS $72 \mathrm{~h}$ after stress conditions, which is possibly related to the excessive activation and dysfunction of the CNS in chronic injuries.
\end{abstract}

\section{Introduction}

Glial cells in the central nervous system (CNS) maintain the homeostasis of the internal environment. Glial cells and especially astrocytes (AS) form glial scars through activation and proliferation. AS secrete a variety of inhibitory factors stimulated by injury, ischemia, blood-brain barrier damage, inflammatory reaction, abnormal metabolism and oxidative stress. These external factors critically affect axon regeneration and recovery of neurological function in the CNS (1). Increased expression of glial fibrillary acidic protein (GFAP) is one of the most important markers of AS activation (2). Culturing neurons and AS together show that local inflammatory response is significantly enhanced by

Correspondence to: Dr Rui Gu, Department of Orthopedics, China-Japan Union Hospital of Jilin University, 126 Xiantai Street, Changchun, Jilin 130033, P.R. China

E-mail: 642403512@qq.com

Key words: astrocytes, glial fibrillary acidic protein, 14-3-3e protein inhibiting GFAP production (3). GFAP inhibition increased neuronal death, suggesting the protective effects of GFAP in the nervous system injury (3). The AS intermediate filament protein system, which includes GFAP, is not only an important component of the cytoskeleton but also a vital signaling system that responds to a variety of stress conditions, and regulates cell size, migration and survival via multiple proteins (4). The $14-3-3$ protein is widely distributed in all tissues and is one of the most abundant proteins in $\mathrm{CNS}$, accounting for $1 \%$ of soluble protein content (5). 14-3-3 proteins are involved in regulating most critical cell activities, including signal transduction, cell cycle regulation, apoptosis, cytoskeleton dynamics, ionic, and protein transmembrane transport, making 14-3-3 a potential therapeutic target for many conditions (6). In this study, we generated an in vivo injury model with AS to study the expression of GFAP and $14-3-3 \varepsilon$.

\section{Materials and methods}

Isolation of rat AS. Generation of AS cultures from newborn Sprague-Dawley rats: Dura mater was cut into small pieces of $1 \mathrm{~mm}^{3}$, preheated medium [10\% fetal bovine serum (FBS), penicillin, high glucose Dulbecco's modified Eagle's medium (DMEM); Biosharp, Hefei, China] was added to obtain AS after pipetting, filtration, centrifugation, discarding the supernatant, cells were placed in $5 \% \mathrm{CO}_{2} 37^{\circ} \mathrm{C}$ incubator, changing the medium once, and sub-cultured after 2 weeks of culture. This study was approved by the Animal Ethics Committee of Jilin University Animal Center.

Main instruments and reagents. Double clean benches (YJ-1450, Boxum; Shanghai Boxun Industry Co. Ltd. Medical Equipment Factory, Shanghai, China), carbon dioxide incubator (HF151UV, Heal Force; Shanghai Li Kang Instrument, Shanghai, China), inverted microscope (XDS-D2; Optical Instrument \& Equipment Factory, Chongqing, China), inverted fluorescence microscope (Zeiss, Oberkochen, Germany), multifunctional microplate analyzer (BioTek Synergy 2; BioTek Instruments, Inc., Winooski, VT, USA), gel electrophoresis system (Chempgpl-5000; Beijing Saizhi Pioneer Technology, Beijing, China), electrophoresis (DYY-6Y) and membrane transfer device (DY-C1) (both from Beijing Liuyi Instrument Factory, Beijing, China), Odyssey double-color infrared fluorescence imager (LI-COR Biosciences, Lincoln, 


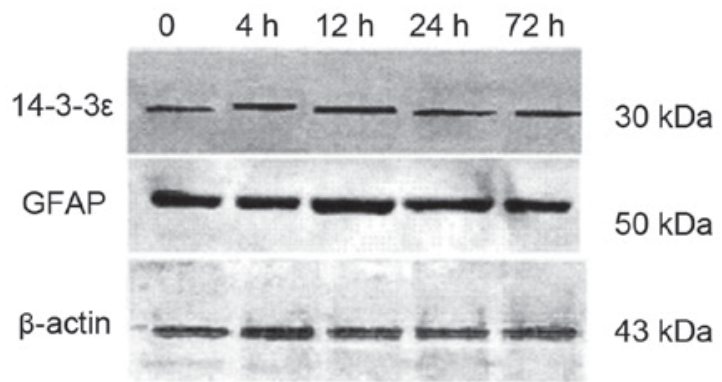

Figure 1. Expression of GFAP and $14-3-3 \varepsilon$ by western blot analysis at different time-points. GFAP, glial fibrillary acidic protein.

NE, USA), shaking incubator shaker (Beijing City Sixty-one Instrument Factory, Beijing, China), pure water (Heal Force Bio-Meditech Holdings Limited, Hong Kong, China).

High-sugar and sugar-free DMEM, Australian FBS, green-streptomycin, and $0.25 \%$ trypsin were purchased from Gibco (Grand Island, NY, USA). BCA protein assay kit (Pierce Biotechnology, Inc., Rockford, IL, USA), nitrocellulose membrane (Hybond; GE Healthcare Life Sciences, Shanghai, China), RIPA lysate (Biyuntian Biotechnology Research Institute, Jiangsu, China). Mouse monoclonal GFAP antibody (dilution, 1:500; cat. no. sc-71143) and mouse monoclonal $\beta$-actin antibody (dilution, 1:500; cat. no. sc-130065) were purchased from Santa Cruz Biotechnology, Inc. (Santa Cruz, CA, USA). Rat anti-mouse IRD-labeled IgG secondary antibodies were purchased from BD Biosciences (Franklin Lakes, NJ, USA) (dilution, 1:1,000; cat. no. 559062) and FITC-labeled goat anti-mouse IgG monoclonal antibody were purchased from Chemicon (Temecula, CA, USA).

Hypoxia/reperfusion (OGD/IR) model. Normal cells (volume, $70-80 \%$ ) were cultured in $2 \mathrm{ml}$ of sugar-free DMEM in a $95 \%$ $\mathrm{N}_{2} / 5 \% \mathrm{CO}_{2}$ hypoxia training box (oxygen concentration was $1 \%$ ), replaced with normal culture medium in a normal culture box $\left(95 \% \mathrm{O}_{2} / 5 \% \mathrm{CO}_{2}\right)$ after $4 \mathrm{~h}$.

Observation index. GFAP and 14-3-3e were detected, respectively, by western blot analysis and double-labeling immunofluorescence detection to test expression level and distribution at 4, 12, 24 and $72 \mathrm{~h}$.

Western blot analysis. After adding lysis buffer, total protein was extracted by conventional methods, sodium dodecyl sulphate-polyacrylamide gel electrophoresis (SDS-PAGE) was completed and proteins transferred to a membrane. Then, washed in TBST, primary antibody and secondary antibodies were added, color was developed, and assessed, and the values were detected semi-quantitatively.

Immunofluorescence. Cells were fixed for $30 \mathrm{~min}$ with $4 \%$ polyformic acid at room temperature, and rinsed with phosphate-buffered saline (PBS) for $5 \mathrm{~min}$, three times; 0.3\% Triton X-100 in PBS was added for 15 min for permeabilization, rinsed in PBS for $5 \mathrm{~min}$, three times, incubated in 3\% BSA for $1 \mathrm{~h}$ covered at room temperature, the primary antibodies (goat anti-GFAP 1:200, goat anti 14-3-3ع 1:100) were added, incubating in a wet box at $4^{\circ} \mathrm{C}$ overnight, rinsed with

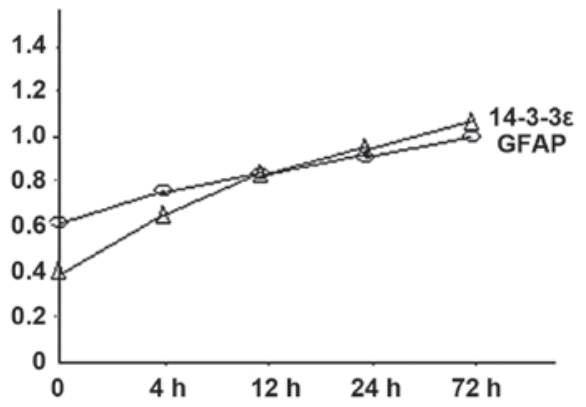

Figure 2. Quantification of western blot analysis data for GFAP and 14-3-3e expression at different time-points. GFAP, glial fibrillary acidic protein.

PBS for $5 \mathrm{~min}$, three times, fluorescent secondary antibody (1:200) was added and incubated for $1 \mathrm{~h}$ at $37^{\circ} \mathrm{C}$ in the dark. Cells were rinsed with PBS for 5 min, three times, incubated in $1 \%$ Hoechst 33258 for 15 min in the dark and evaluated by fluorescence microscopy.

Statistical analysis. We used SPSS 19.0 statistical software (IBM SPSS, Armonk, NY, USA) for data analysis. Quantitative data are expressed as mean \pm standard deviation, using variance analysis of repeated measure data, $\mathrm{P}<0.05$ was considered to indicate a statistically significant difference.

\section{Results}

Expression levels of GFAP and 14-3-3e in hypoxic AS. We isolated rat $\mathrm{AS}$ and placed them in hypoxic culture conditions. Then, we examined the expression of GFAP and 14-3-3 $\varepsilon$ at 4, 12, 24 and $72 \mathrm{~h}$. Western blot analysis showed that expression of both GFPA and 14-3-3e progressively increased over time (Figs. 1 and 2) and the differences were statistically significant $(\mathrm{P}<0.05)$.

Immunofluorescence localization. We next examined the expression and distribution of GFAP and $14-3-3 \varepsilon$ under the microscope at different time-points. Despite the lower sensitivity of immunofluorescence compared to western blot analysis, we detected the upregulation of both GFAP and 14-3-3e starting at $4 \mathrm{~h}$ following hypoxia and increasing until $72 \mathrm{~h}$ (Fig. 3). GFAP and 14-3-3e co-localized mainly in the cytoplasm of AS (Fig. 3).

\section{Discussion}

Our studies suggest that expression of GFAP and 14-3-3e increase with time during hypoxia, and that GFAP and 14-3-3e co-localize in the cytoplasm in cultured AS. AS are involved in most critical aspects of CNS function.i) AS provide support and energy nutrition for neuronal survival, development, differentiation, and regeneration (7). ii) AS contribute to neurotransmitter uptake and degradation, regulating the synaptic connections, and signal transduction (8). iii) AS have immune activity, expressing signal of MHCII molecules and co-stimulatory, and participating in antigen presentation and activation of T cells (9). iv) AS play an important role in the formation of the endothelial barrier, maintaining their morphology, releasing a variety of chemical substances, 


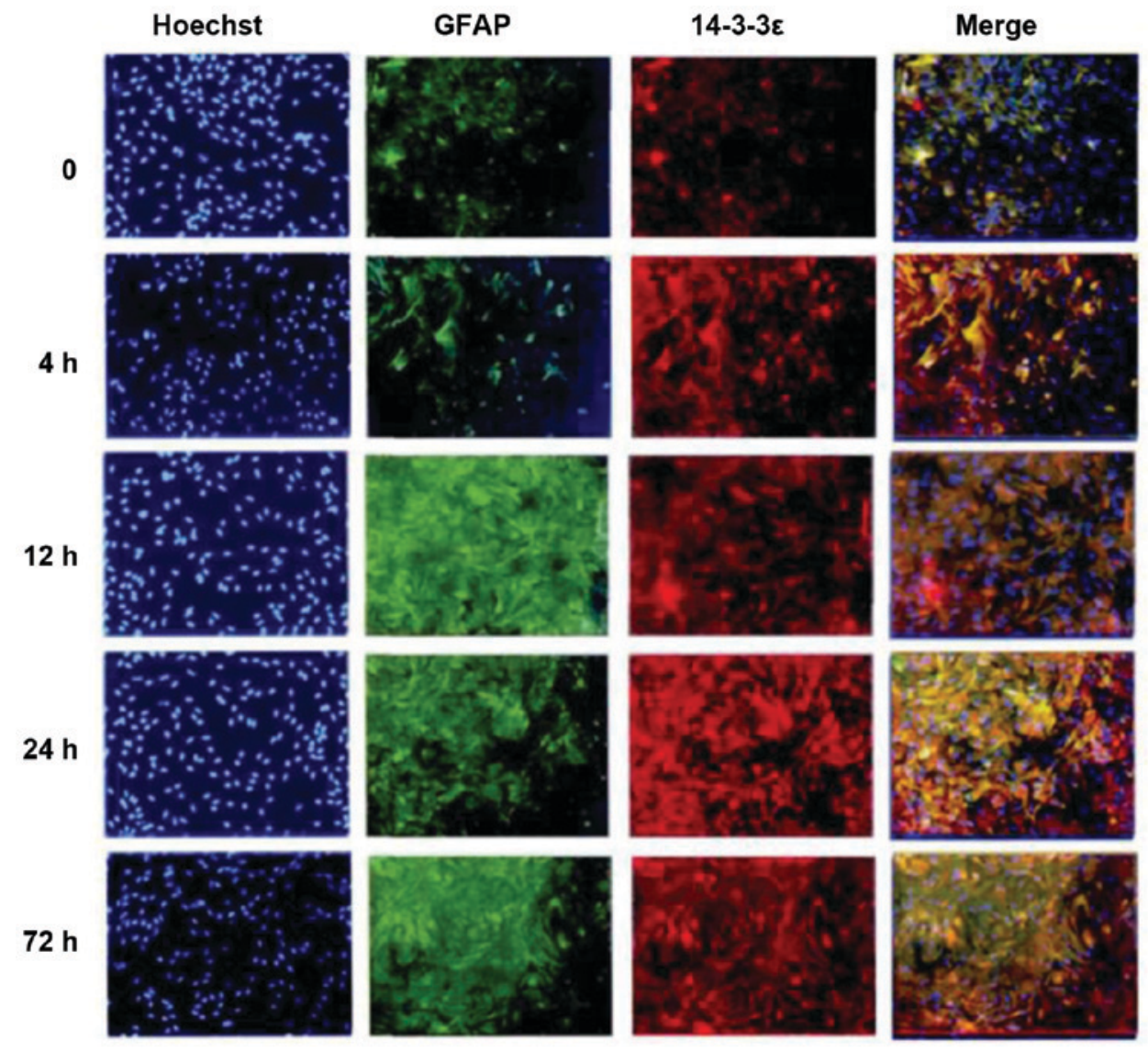

Figure 3. Double-labeling immunofluorescence of GFAP and 14-3-3e in astrocytes subjected to hypoxia for different times. GFAP (green), 14-3-3e (red), and Hoechst (blue). All images were collected at x400 magnification. GFAP, glial fibrillary acidic protein.

and regulating the permeability of endothelia cells as an important component of CNS blood-brain barrier (10). v) AS regulate the immune inflammatory reaction of CNS by secreting cytokine and inflammatory factors, thereby contributing complex behavior of the whole body, such as pain, appetite, sleep and mood through the circulatory system (11). AS can also clear the excitatory glutamate and potassium ions at the site of injury and play a protective role in the survival of neurons (12). AS can secrete Wnt3, interleukin (IL)-1, IL-6, insulin-like growth factor binding protein 6 , and other substances to promote the colonization of stem cells, providing a favorable microenvironment for nerve regeneration (13).

GFAP is an intermediate filament protein with a diameter of 8-9 nm that is present mainly in AS. GFAP has no special contribution in the normal nervous system, but plays a vital role in the glial scar and the activation of AS. Activation of AS and expression of GFAP inhibits inflammatory response after injury, effectively limiting the damage within a controllable region (14). Treatment with the dopaminergic neurotoxin MPTP selectively damaged the nerve endings and started AS activation, which induced and promoted transcription and translation of GFAP by JAK/STAT3 signaling pathway (15). The expression of GFAP and vimentin was inhibited in $\mathrm{GFAP}^{-/-}$and $\mathrm{Vim}^{-/}$transgenic mice, which demonstrated that infarct area after ischemic stroke in transgenic mice were significantly increased compared with the control group. In the ischemia injury GOD model, generation and accumulation of ROS increased significantly in transgenic group, resulting in more nerve cell death, suggesting that GFAP was particularly important to reduce oxidative stress injury in the acute phase of the cells (16). With the continued progress of the injury and AS activation, GFAP expression increased progressively becoming an important factor in promoting glial cicatrization, although it was not conducive in restoring nerve function and nerve regeneration (17). How to effectively take advantage of GFAP protective role in early response to damage, while finding the right time to inhibit its activity to prevent the formation of glial scar, are important questions for future study.

Inflammation, vascular dementia, cancer, metabolic diseases, epilepsy, mental disorders, and other nervous system disorders lead to abnormal expression of 14-3-3 in the cerebrospinal fluid. For this, 14-3-3 is considered a marker of damaged nerve cells and its level of expression may be related to the extent of injury (18). 14-3-3e binding to the multiple molecules of the Ras/MAPK signaling pathway has critical functions as a cytoskeletal protein and regulates protein synthesis, metabolism, DNA damage and repair, and the cell cycle (19). Chronic high expression of 14-3-3e during injury inhibits AS apoptosis after injury (20) and promotes the activation of AS by inflammation reaction in early response through the ERK1/2 and $\mathrm{NF}-\kappa \mathrm{B}$ signaling pathways (21). In addition, activated MAPK pathway secretes TNF- $\alpha$, IL-1 $\beta$, and other inflammatory mediators, indirectly activates the JAK/STAT3 pathway, promotes AS activation and secreting GFAP, and many other 
inflammatory factors, which increase the inflammatory immune response to damage, further altering the function of synapses and neurons (22).

\section{Acknowledgements}

The present study was supported by the National Natural Science Foundation of China, no. 81301051.

\section{References}

1. Sofroniew MV: Molecular dissection of reactive astrogliosis and glial scar formation. Trends Neurosci 32: 638-647, 2009.

2. Pekny M and Pekna M: Astrocyte intermediate filaments in CNS pathologies and regeneration. J Pathol 204: 428-437, 2004.

3. Lefrançois T, Fages C, Peschanski M and Tardy M: Neuritic outgrowth associated with astroglial phenotypic changes induced by antisense glial fibrillary acidic protein (GFAP) mRNA in injured neuron-astrocyte cocultures. J Neurosci 17: 4121-4128, 1997.

4. Pekny M and Lane EB: Intermediate filaments and stress. Exp Cell Res 313: 2244-2254, 2007.

5. Yaffe MB: Phosphotyrosine-binding domains in signal transduction. Nat Rev Mol Cell Biol 3: 177-186, 2002.

6. Tinti M, Johnson C, Toth R, Ferrier DE and Mackintosh C: Evolution of signal multiplexing by 14-3-3-binding 2R-ohnologue protein families in the vertebrates. Open Biol 2: 120103, 2012.

7. Parpura V, Heneka MT, Montana V, Oliet SH, Schousboe A, Haydon PG, Stout RF Jr, Spray DC, Reichenbach A,PannickeT, et al: Glial cells in (patho)physiology. J Neurochem 121: 4-27, 2012.

8. Verkhratsky A: Physiology of neuronal-glial networking. Neurochem Int 57: 332-343, 2010.

9. VanGuilder HD, Bixler GV, Brucklacher RM, Farley JA, Yan H, Warrington JP, Sonntag WE and Freeman WM: Concurrent hippocampal induction of MHC II pathway components and glial activation with advanced aging is not correlated with cognitive impairment. J Neuroinflammation 8: 138, 2011.

10. Herland A, van der Meer AD, FitzGerald EA, Park TE, Sleeboom JJ and Ingber DE: Distinct contributions of astrocytes and pericytes to neuroinflammation identified in a 3D human blood-brain barrier on a chip. PLoS One 11: e0150360, 2016.

11. Ingiosi AM and Opp MR: Sleep and immunomodulatory responses to systemic lipopolysaccharide in mice selectively expressing interleukin-1 receptor 1 on neurons or astrocytes. Glia 64: 780-791, 2016.
12. Navarrete $M$ and Araque A: Endocannabinoids mediate neuron-astrocyte communication. Neuron 57: 883-893, 2008.

13. Barkho BZ, Song H, Aimone JB, Smrt RD, Kuwabara T, Nakashima K, Gage FH and Zhao X: Identification of astrocyte-expressed factors that modulate neural stem/progenitor cell differentiation. Stem Cells Dev 15: 407-421, 2006.

14. Cheon SY, Cho KJ, Song J and Kim GW: Knockdown of apoptosis signal-regulating kinase 1 affects ischaemia-induced astrocyte activation and glial scar formation. Eur J Neurosci 43: 912-922, 2016

15. Sriram K, Benkovic SA, Hebert MA, Miller DB and O'Callaghan JP: Induction of gp130-related cytokines and activation of JAK2/STAT3 pathway in astrocytes precedes up-regulation of glial fibrillary acidic protein in the 1-methyl-4-phenyl-1,2,3,6-tetrahydropyridine model of neurodegeneration: key signaling pathway for astrogliosis in vivo? J Biol Chem 279: 19936-19947, 2004.

16. de Pablo Y, Nilsson M, Pekna M and Pekny M: Intermediate filaments are important for astrocyte response to oxidative stress induced by oxygen-glucose deprivation and reperfusion. Histochem Cell Biol 140: 81-91, 2013.

17. García-Álvarez I, Fernández-Mayoralas A, Moreno-Lillo S, Sánchez-Sierra M, Nieto-Sampedro M and Doncel-Pérez E: Inhibition of glial proliferation, promotion of axonal growth and myelin production by synthetic glycolipid: a new approach for spinal cord injury treatment. Restor Neurol Neurosci 33: 895-910, 2015.

18. Foote $\mathrm{M}$ and Zhou Y: 14-3-3 proteins in neurological disorders. Int J Biochem Mol Biol 3: 152-164, 2012.

19. Lalle M, Leptourgidou F, Camerini S, Pozio E and Skoulakis EM: Interkingdom complementation reveals structural conservation and functional divergence of 14-3-3 proteins. PLoS One 8: e78090, 2013.

20. Wachi T, Cornell B, Marshall C, Zhukarev V, Baas PW and Toyo-oka K: Ablation of the 14-3-3gamma protein results in neuronal migration delay and morphological defects in the developing cerebral cortex. Dev Neurobiol 76: 600-614, 2016.

21. Yan L, Gu H, Li J, Xu M, Liu T, Shen Y, Chen B and Zhang G: RKIP and $14-3-3 \varepsilon$ exert an opposite effect on human gastric cancer cells SGC7901 by regulating the ERK/MAPK pathway differently. Dig Dis Sci 58: 389-396, 2013.

22. Nishino T, Matsunaga R and Konishi H: Functional relationship between CABIT, SAM and 14-3-3 binding domains of GAREM1 that play a role in its subcellular localization. Biochem Biophys Res Commun 464: 616-621, 2015. 